\title{
Humanización
}

\section{La espiritualidad en la humanización de la asistencia sanitaria}

Spirituality in the Humanization of Health Assistance

8) Autores

\author{
David Lorenzo \\ Campus Docent Sant Joan de Déu \\ E-mail: dlorenzo@santjoandedeu.edu.es \\ http://orcid.org/0000-0002-7647-4224
}




\section{Resumen}

Abstract
La consideración de la dimensión espiritual del ser humano es un elemento importante para humanizar la asistencia sanitaria. En las últimas décadas, esa dimensión se ha ido incorporando a la atención sanitaria, de modo que, en la actualidad, el modelo de atención puede considerarse un modelo "bio-psico-social-espiritual". No obstante, dicha dimensión suele tratarse desde la perspectiva de la necesidad, una mirada que tiende a centrarse en las carencias del individuo. La espiritualidad también puede verse como ámbito de capacidades, de recursos para afrontar mejor la enfermedad. En este artículo, se pretende mostrar que la consideración de la espiritualidad como fuente de capacidades es un elemento importante para la humanización de la asistencia.

The consideration of the spiritual dimension of human beings is an important element in order to humanize health care. In recent decades this dimension has been incorporated into health care in such a way that, at present, the model could be considered as a "bio-pyscho-social-spiritual" model. However, said dimension tends to be dealt with from the perspective of need, a view that tend to focus on the needs of the individual. Spirituality can also be seen as a field of capabilities, of resources to better deal with the illness. This paper aims to portray that the consideration of spirituality as a source of capabilities is an important element for the humanization of health care.

Humanización; asistencia sanitaria; espiritualidad; necesidades espirituales; capacidades espirituales.

Humanization; health assistance; spirituality; spiritual needs; spiritual abilities. 


\section{Introducción: espiritualidad y humanización}

Es un tópico la afirmación de que la asistencia sanitaria está deshumanizada. En muchas ocasiones, detrás de dicha afirmación, se haya la constatación de que esta no cumple unos mínimos considerados imprescindibles para preservar la dignidad de la persona (del paciente), o bien la sensación de que tales mínimos se cumplen, pero sin alcanzar unos estándares de calidad idóneos: la asistencia cumple unas condiciones necesarias, pero no otras que, aunque no necesarias, sí pueden considerarse deseables o convenientes.

"Cuando se habla de humanizar la atención en salud, se hace referencia en principio, a todas aquellas acciones que hacen más humano al hombre..." (Correa, 2016, p. 1227).

En esa misma línea, se puede considerar que humanizar la asistencia (o cualquier otro acto o realidad) es el proceso o el acto

Comprender la enfermedad implica comprender a la persona enferma, y comprender

a la persona enferma

implica comprender todas

sus dimensiones: la física,

la psicológica, la social y la

espiritual por el cual algo deviene o se hace humano (o más humano), o también - podría añadirse- digno de la persona, es decir, acorde con las exigencias de la condición humana (Vielva, 2007, p. 223).

Esta condición -la humana- tiene unas características específicas que la distinguen de la condición de otros seres (objetos, vegetales y animales). Entre ellas, destaca, a nuestro juicio, la espiritualidad. Y destaca porque precisamente es el espíritu humano -el alma humana- el que dota al ser humano de unas facultades (inteligencia y voluntad) que permiten al hombre una serie de operaciones que ningún otro ser en la naturaleza puede realizar.

La dimensión espiritual del ser humano - del paciente- ha sido objeto de creciente atención e interés en las ciencias de la salud. Durante las últimas décadas, estas han ido ampliando progresivamente su visión del hombre y la enfermedad. La asistencia sanitaria ha evolucionado desde un modelo "biologicista" hacia un modelo "bio-psico-social". En los últimos años, ese modelo se ha ampliado y ha incluido una nueva dimensión: la espiritual. Comprender la enfermedad implica comprender a la persona enferma y comprender a la persona enferma implica comprender todas sus dimensiones: la física, la psicológica, la social y la espiritual.

Correa, basándose en diversos autores (Bermejo-Higuera, Arredondo, Montealegre, etc.), señala:

La humanización de la salud apunta al objetivo central de orientar la actuación en el mundo de la salud hacia el servicio de la persona enferma, considerándolo en su globalidad personal y tratando de ofrecerle una asistencia integral con calidez, que responda a las dimensiones psicológica, biológica, social y espiritual. (Correa, 2016, p. 1228)

En la misma línea, afirma Vielva (2007, pp. 226-227) que la humanización tiene dos facetas o vertientes: la que se refiere al personal sanitario y la que se refiere al pa- 
ciente. Dentro de la humanización que tiene por objeto el paciente, distingue dos tipos de factores: los externos y los internos. Los externos se refieren a la visión de la profesión que de sí misma puede tener la enfermería (o la medicina), a las condiciones económicas, materiales y administrativas que enmarcan el ejercicio de la asistencia, etc. Los internos se refieren a la relación entre el personal asistencial y el paciente, al reconocimiento del valor de la persona (aunque esté en una situación vulnerable) y al reconocimiento y atención de sus necesidades, necesidades que son muy diversas: emocionales, psicológicas, espirituales, etc.

Acabamos de hablar de "necesidades"... En la actualidad, la asistencia sanitaria, ciertamente, ha incorporado -va incorporando- a su atención la dimensión espiritual de la persona. No obstante, el tratamiento de esa dimensión se ha hecho desde una perspectiva muy determinada: la de la necesi-

La dimensión espiritual del paciente puede también considerarse como fuente de capacidades, de

potencialidades, de recursos para afrontar mejor la vida y la enfermedad dad. La literatura médica y enfermera suele hablar de "atender" las necesidades espirituales del paciente, de "medir" o "evaluar" esas necesidades, de diseñar instrumentos para "detectarlas", etc. Las diversas acciones o estrategias para considerar esa dimensión se dirigen siempre a la necesidad, es decir, a cubrir una carencia. Pero la dimensión espiritual del paciente puede también considerarse como fuente de capacidades, de potencialidades, de recursos (para afrontar mejor la vida y la enfermedad). En este artículo, pretendemos mostrar la relación entre espiritualidad y humanización a través del concepto de "capacidad".

\section{Espíritu y trascendencia}

La inteligencia y la voluntad, facultades clásicamente presentadas como las propias del ser humano, son facultades trascendentes. Una cualidad esencial del espíritu es la trascendencia. Para R. M. Nogués (2013, pp. 9-10), la trascendencia es la cualidad esencial de la mente humana, lo que hace al hombre lo que es. Es una cualidad que permite acciones no necesarias desde el punto de vista biológico. "Trascender significa ir más allá de la realidad inmediata y, concretamente, más allá de las necesidades estrictas" (Nogués, 2013, p. 84). El ser humano es cuerpo, pero no solo eso. "Sólo el hecho de reflexionar sobre ello señala un campo que le sitúa en un plano radicalmente distinto" (Torralba, 2016, p. 72). La libertad depende de esa distancia: radica en la posibilidad de liberarse de los dictados del cuerpo. "No podemos dejar de ser cuerpo, pero podemos ser más que cuerpo" (Torralba, 2016, p. 72).

Esa idea es la que está detrás del informe de la OMS sobre cuidados paliativos y cáncer cuando afirma que lo espiritual "se refiere a esos aspectos de la vida humana relacionados con experiencias que trascienden los fenómenos sensibles" ("... refers to those aspects of human life relating to experiences that trascend sensory phenomena") (OMS, 1990, p. 50). Por eso afirma Torralba que lo espiritual es "lo libre del ser humano, lo que escapa a lo biológico, aunque esté intrínsecamente unido a lo somático" (Torralba, 2016, p. 54). 
La dimensión espiritual permite al ser humano distanciarse de su biología e impulsos y, a la vez, salir de/transcender su "ego" (Torralba, 2016, p. 54). El espíritu, pues, permite al hombre "abandonar" su fisicidad (su corporeidad) y distanciarse de todo: del mundo, de lo que le rodea y de sí mismo (Frankl, 1994, p. 110). Por ello afirma Torralba que el ser humano puede ser actor y espectador a la vez, mientras que los animales y las plantas no pueden sino actuar, ser solo actores (Torralba, 2016, p. 93)'1.

Aunque la dimensión espiritual del hombre se ha añadido al modelo "bio-psico-social" y ha sido objeto de interés práctico y teórico, la presencia y consideración de dicha dimensión en el ámbito clínico sigue siendo, en general, escasa.

\section{Espiritualidad: necesidad y capacidad}

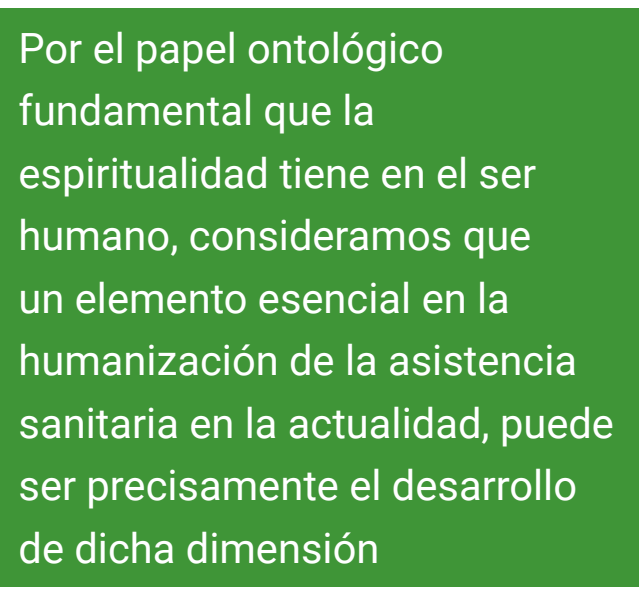

Por el papel ontológico fundamental que la espiritualidad tiene en el ser humano, consideramos que un elemento esencial en la humanización de la asistencia sanitaria en la actualidad puede ser precisamente el desarrollo de dicha dimensión. En ese sentido, su tratamiento puede ser abordado desde dos perspectivas: desde la necesidad y desde la capacidad. La primera (la necesidad) es un abordaje -creemos- de tipo más bien "pasivo", mientras que el segundo (la capacidad) supone un acercamiento más bien "activo".

Basamos dicha distinción en el abordaje que la literatura nos ofrece. La literatura médico-enfermera muestra, en general, que la consideración de la dimensión espiritual del paciente ha cristalizado de dos modos, según esas dos tendencias: por una parte, en una vertiente centrada en el concepto de necesidad; por otra, en una vertiente centrada en el concepto de capacidad. La vertiente basada en la necesidad pone atención a las carencias o vacíos del paciente en el ámbito de la espiritualidad, para detectarlas y evaluarlas a través de instrumentos y escalas de medición, cubrirlas, etc. La vertiente basada en la capacidad pone atención en los resortes o poderes espirituales que el paciente tiene para afrontar mejor la enfermedad.

Ambas facetas o vertientes se aprecian, por ejemplo, en la guía El acompañamiento espiritual en cuidados paliativos de la SECPAL:

No entendemos por "necesidades espirituales" únicamente la acepción de déficit, carencia o vacío no cubierto; también nos referimos a aquellas potencialidades aún no suficientemente desarrolladas o a aquellas expectativas no suficientemente cubiertas, pero sí deseadas, en el ámbito de lo espiritual. Esta clave es importante, porque nos sitúa ante la realidad espiritual del ser humano al final de la vida no

1 Conviene señalar, sin embargo, que, aunque se hable aquí de dos ámbitos distintos (lo espiritual y lo corporal), consideramos la persona como una unidad sustancial, no una suma accidental de dos realidades o entidades separadas. 
sólo desde el abordaje de la amenaza (las carencias), sino también desde la óptica de la oportunidad (los recursos aún no suficientemente empleados o desarrollados). (SECPAL, 2008, p. 52)

La misma línea siguen J. Barbero, X. Gómez-Batiste, J. Maté y D. Mateo, coordinadores del Manual para la atención psicosocial y espiritual a personas con enfermedades avanzadas, al hablar de la importancia de tener en cuenta las capacidades del paciente (no se refieren específicamente a las espirituales):

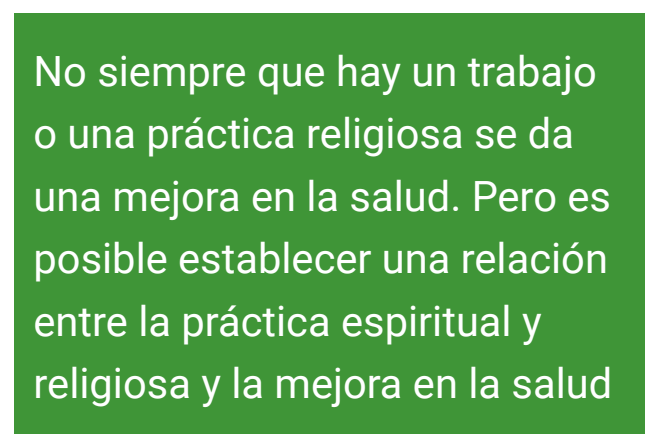

Habitualmente, de los pacientes conocemos fundamentalmente sus dificultades, sus problemas, sus signos y síntomas, es decir, todo aquello que los describe en sus heridas [...]. En la universidad no se nos forma para detectar capacidades, recursos, potencialidades, etc. Esa parte es la que ponemos nosotros, los profesionales, desde nuestra formación, experiencia y buena voluntad. Ellos ponen las heridas, y nosotros, las capacidades sanadoras. Este enfoque se encuentra en la base del modelo paternalista, enormemente beneficentista [...]. Los pacientes aportan a la relación terapéutica, obviamente, sus heridas: enfermedad de base, recidivas, incertidumbre, angustia, familia descolocada, multitud de pérdidas y un largo etcétera. Pero también nos aportan sus capacidades sanadoras, sus recursos, sus potencialidades. Es decir, el apoyo familiar, estrategias de afrontamiento más o menos potentes, su formación, sus redes sociales, su espiritualidad, su historia biográfica y también un largo etcétera. (Barbero, Gómez-Batiste, Maté y Mateo, 2016, p. 80)

Las ciencias de la salud, llevadas por la intención noble de ofrecer una atención holística, han incluido y se han acercado a la dimensión espiritual del paciente desde el prisma de la necesidad, adoptando la misma mirada desde la que se abordan otras dimensiones, especialmente la biológica, la fisiológica. Ese abordaje -creemos- ha dejado en un segundo plano una visión más "activa" de la espiritualidad del paciente, una visión que atienda también a la capacidad².

Ese abordaje "activo" de la espiritualidad, si bien poco desarrollado o atendido en la literatura, no es extraño a las investigaciones del ámbito de las ciencias de salud. Según Hemann, son muchos los estudios que permiten afirmar que "la religión y la espiritualidad han mostrado ayudar a las personas a afrontar la enfermedad y la muerte" (Hermann, 2006, p. 737). Es verdad que los resultados no siempre van en la misma línea ni confirman esa conclusión: no siempre que hay un trabajo o una práctica religiosa se

2 Hemos hablado de la inteligencia y la voluntad como las facultades propias del espíritu. Las capacidades en que se pueden concretar dichas facultades son muy diversas y pueden ser objeto de discusión y debate. No es posible aquí ahondar en la definición y en la delimitación de ellas. La lista es variable en función de los autores. F. Torralba, por ejemplo, señala 17 poderes espirituales (él habla, en concreto, de los "poderes de la inteligencia espiritual"), de los que destacamos estos: la búsqueda de sentido, la facultad de valorar, la capacidad de distanciamiento, el asombro, el gozo estético, el autoconocimiento, la capacidad de religación y la elaboración de ideales de vida (Torralba, 2016, p. 8). 
da una mejora en la salud (aunque sea en aspectos de detalle como, por ejemplo, la hipertensión) (Alpert, 2010, p. 141; Hermann, 2006, p. 737). Pero es posible establecer una relación entre la práctica espiritual y religiosa y la mejora en la salud (Aukst-Margetic y Margetic, 2005; Stefanek, McDonald, y Hess, 2005; Hermann, 2006).

P. T. Alpert, en su artículo "Spirituality Goes Beyond Religiosity: A Much Needed Practice in Nursing" (2010), cita diversos estudios que demuestran que las diferentes prácticas espirituales y religiosas (orar, meditar, la vida de fe, etc.) influyen positivamente en la salud de las personas. Algunos ejemplos son estos: la práctica religiosa puede asociarse con la reducción de los síntomas de depresión, ansiedad y estrés (estudio de Ano y Vasconcelles, 2005); la probabilidad de sobrevivir a determinadas enfermedades es un $29 \%$ más alta en personas con alguna práctica espiritual habitual (investigación de McCullough, Larson, Hoyt, Koening y Thoresen, 2000); el sistema inmunitario en un grupo de personas con VIH es más fuerte en aquellas con un cuidado espiritual o religioso (estudio de Pargament, McCarthy, Shah, Ano, Tarakeshwar, y Wachholtz, 2004); la práctica de la oración mejora la salud en pacientes con una enfermedad coronaria y ayuda a aumentar los porcentajes de supervivencia en niños con leucemia (estudio de Townsend, Kladder, Ayele, y Mulligan, 2002) (Alpert, 2010, p. 141).

Pensamos que los datos que arrojan esas investigaciones vienen a ser ejemplos de cómo las capacidades espirituales del ser humano pueden influir en la salud de un individuo cuando se desarrollan y se ejercitan. Muestran que el ámbito de la espiritualidad del hombre puede ser objeto tanto de necesidad como de capacidad, tanto de carencia como de potencialidad.

\section{El espíritu ante la enfermedad}

Es clásica la distinción que la antropología de la salud establece entre tres vertientes de la enfermedad: disease, illness y sickness. Disease se refiere a la vertiente biológica, fisiológica o técnica de la enfermedad. Illness refleja la vivencia subjetiva de disease, es decir, cómo el individuo, partiendo de los parámetros conceptuales de su cultura y de su pensamiento propio, interpreta, vive y experimenta la disease que padece. Sickness significa o expresa la vertiente social y política de la enfermedad, cómo esta influye en la relación de la persona enferma con los demás, con la sociedad, con el cuerpo político, etc. (Bouché, 2001, p. 62; Comelles y Martínez, 1993, pp. 61-62; 85-87; Helman, 2001, pp. 79-84).

Las capacidades espirituales pueden relacionarse con las tres dimensiones de la enfermedad, pero especial o principalmente con illness. La búsqueda de sentido, el distanciamiento, la elaboración de ideales de vida, etc., condicionan de manera determinante y fundamental la vivencia de la enfermedad por parte del paciente. 
Un ejemplo de ello son las distintas visiones o interpretaciones que, partiendo de su herencia cultural, el individuo puede tener sobre la enfermedad y el sufrimiento. La Carta de identidad de la Orden Hospitalaria de San Juan de Dios señala cinco (OHSJD, 2012, pp. 29-30).

a) Concepción mágica o misteriosa. En ella, el sufrimiento se concibe como un castigo o como una manifestación de un ser u orden sobrenatural. Los remedios pueden ser, por ello, también sobrenaturales. El chamanismo y la religiosidad de tribus primitivas son muestras paradigmáticas de esta concepción.

b) Negación. Desde esta perspectiva, el sufrimiento es límite y negación de la vida y del placer, por lo que el hombre debe huir de él y aprovechar el placer o bienestar mientras pueda. Esta visión conecta con concepciones filosóficas de corte epicureísta, hedonista o nietzscheano.

c) Aceptación. Para esta visión, el sufrimiento se considera algo irremediable que forma parte del destino (también irremediable) y que exige del hombre racionalización y entereza para acogerlo. Tal acercamiento se relaciona con una antropología de base estoica.

d) Anulación. Según esta concepción, el sufrimiento se puede mitigar, acallar o acabar tras un proceso disciplinado por parte del individuo de abandono y anulación de todo deseo y pasión. El budismo sería un ejemplo de esta posición.

e) Valorización. El sufrimiento, para esta posición, puede ser "reconvertido" y recibir un valor como medio para hacer el bien al individuo y a la humani-

Conviene señalar que la relación

capacidad-enfermedad no

es unidireccional: no solo

influyen las capacidades en

la enfermedad, sino que esta

influye en aquellas dad. El cristianismo es el paradigma de esta concepción.

Aunque la influencia de las capacidades espirituales se dé sobre todo en "illness", estas también influyen -como se ha dicho- en disease y sickness. Con respecto a disease, ya hemos visto que hay estudios que demuestran que el desarrollo habitual de actividades espirituales puede tener influencia sobre determinados procesos de una enfermedad o el curso de la misma (influyendo en factores como el sistema inmunitario, el estrés, la hipertensión, la depresión, etc.).

Con respecto a sickness, las capacidades espirituales -el modo y el grado en que se ejerzan- pueden influir en las relaciones sociales y políticas del individuo. Una enfermedad, por ejemplo, puede llevar al individuo al alejamiento afectivo y relacional de los demás (tendencia natural en muchas ocasiones), pero también al acercamiento a ellos y a establecer relaciones más armónicas que antes. La fuerza transformadora de las capacidades espirituales del ser humano es grande y, en muchas ocasiones, sorprendente.

A la vez, conviene señalar que la relación capacidad-enfermedad no es unidireccional: no solo influyen las capacidades en la enfermedad, sino que esta influye -puede influir- en aquellas. La enfermedad, por su impacto global en la vida del individuo, por ser un hecho biográfico y no solo biológico y, a causa de ello, poder constituir una situación límite, puede transformar las capacidades espirituales del individuo de una manera pro- 
funda. Que el individuo, ante una enfermedad -especialmente si es grave- replantee sus valores, su modo de ver la realidad y la vida, su relación con los demás, su mirada..., son muestras de esa bidireccionalidad.

Lo que venimos describiendo (vertientes de la enfermedad, bidireccionalidad, etc.) se basa en la naturaleza espiritual del ser humano. Por tener esa naturaleza, el hecho de la enfermedad, el "suceso enfermedad" -en palabras del antropólogo A. Zempléni (Bouché, 2001, p. 64) - tiene una doble manifestación en el individuo y un doble modo de percepción por su parte: el dolor y el sufrimiento. La clásica distinción entre "dolor" y "sufrimiento" refleja, a nuestro juicio, esos dos niveles de manifestación y de percepción: un hecho (el "suceso enfermedad") ocurre o se manifiesta y se "experimenta" en dos esferas o niveles.

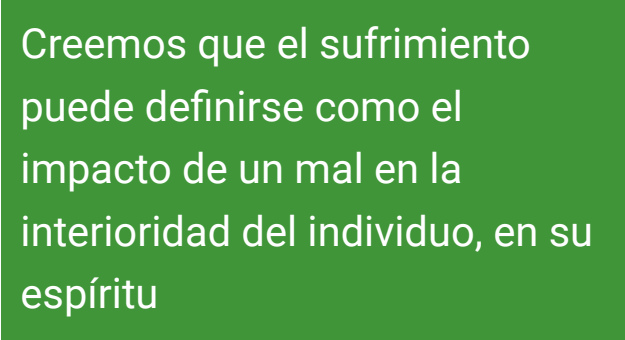

El dolor es una sensación corporal, es un fenómeno corporal que, en general, sirve de señal o de aviso de que un órgano o de que una parte del cuerpo está amenazada. Los seres sintientes perciben esa información como medida de protección (Yepes y Aranguren, 1998, pp. 322-325). Por el contrario, el sufrimiento es un fenómeno más "global" o "etéreo" -si se permite la expresión-. Es una percepción no sensitiva, aunque pueda tener manifestaciones o efectos sensibles. Por ello, no está localizada en un punto o una parte concreta del cuerpo y, a la vez, es percibida. Esa percepción se da en el ámbito de lo que se denomina habitualmente "interioridad" de la persona.

En nuestra opinión, el sufrimiento no es la mera vivencia de un dolor o enfermedad o un desajuste de tipo emocional o psicológico. Creemos que el sufrimiento puede definirse como el impacto de un mal en la interioridad del individuo, en su espíritu. No es este el lugar para ahondar en la definición, pero creemos que indicarla es útil para comprender la naturaleza del sufrimiento y su esencial diferenciación del dolor.

En ocasiones, al menos en determinados estadios, puede existir enfermedad sin dolor y esta causa sufrimiento igualmente. De igual modo, puede existir enfermedad con dolor pero sin sufrimiento (o poco sufrimiento). A la vez, el sufrimiento puede provenir de factores que no son una enfermedad o un dolor: desde un problema económico o laboral grave (indigencia, paro), hasta pequeños problemas o inconvenientes cotidianos (suspender un examen, perder el autobús, etc.). Dichos factores pueden ser más o menos graves, pero todos tienen en común la consideración de "males" por parte del sujeto.

Las capacidades espirituales del individuo tienen su "campo" propio o directo de actuación e influencia en el sufrimiento, no -o no en igual medida- en el del dolor. Ese campo, a diferencia de la esfera del dolor, es amplio, complejo, inaprehensible, lleno de entresijos y pliegues, muchos de ellos desconocidos para el propio el individuo. En ese sentido, la enfermedad puede ser medio de conocimiento -propio y de la naturaleza humana en general-, no a través del dolor -o no tanto- sino a través del sufrimiento. Al experimentar el dolor $y$, en concreto, el sufrimiento que este provoca, la persona -el paciente- puede, a través de su espíritu (de la capacidad de autoconocimiento de la 
inteligencia) "volverse" sobre sí mismo y "posicionarse" ante él en función del sentido de su vida y de sus valores ${ }^{3}$.

\section{Conclusión}

Hemos visto que la espiritualidad es una dimensión esencial del ser humano y que ha sido progresivamente atendida y tenida en cuenta por la asistencia y las ciencias de salud. Esa aceptación ha tenido dos vertientes o modos de acercamiento: desde la necesidad y desde la capacidad. La perspectiva basada o centrada en la necesidad ha sido la predominante. En la literatura médico-enfermera, la espiritualidad del paciente suele aparecer como elemento de humanización "pasivo", es decir, como elemento que requiere "ser cubierto", como una esfera de carencias.

Dicha perspectiva es, ciertamente, importante para ofrecer al enfermo una atención holística y, así, humanizar la asistencia por parte de los profesionales de la salud. Sin embargo, la espiritualidad del paciente puede ser también fuente de capacidades, capacidades que pueden desarrollarse y cuyo ejercicio tiene gran influencia en el abordaje de la enfermedad, del dolor y del sufrimiento por parte del paciente.

Creemos, pues, que ahondar en la espiritualidad del ser humano puede contribuir poderosamente a la humanización de la asistencia y, en concreto, ahondar en ella desde una perspectiva "activa", desde una perspectiva que integre tanto la necesidad como la capacidad.

\section{Bibliografía}

Alpert, P. T. (2010). Spirituality Goes Beyond Religiosity: A Much Needed Practice in Nursing. Home Health Care Management \& Practice, 22(2), 140-143. DOI: https://doi.org/10.1177/1084822309343867

Aukst-Margetic, B., Margetic, B. (2005). Religiosity and health outcomes: Review of literature. Collaborative Anthropology, (29), 365-371.

Barbero, J., Gómez-Batiste, X., Maté, J., y Mateo, D. (Coords.). (2016). Manual para la atención psicosocial y espiritual a personas con enfermedades avanzadas. Barcelona: La Caixa.

Bouché, H. (2001). La salud en las culturas. Educación XX1, 4, 61-90.

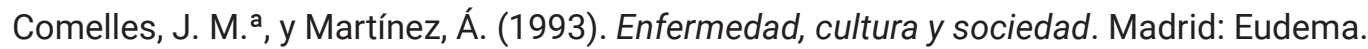

Correa, M. L. (2016). La humanización de la atención en los servicios de salud: un asunto de cuidado. Rev Cuid., 7(1), 1227-31. DOI: http://dx.doi.org/10.15649/cuidarte.v7i1.300

Frankl, V. (1994). El hombre doliente. Barcelona: Herder.

3 Afirma Frankl que siempre el hombre puede conservar un vestigio de libertad - "la última de las libertades humanas"- aunque le arrebaten todo: la elección de la actitud personal ante las circunstancias, ante el destino (Frankl, 2010, p. 90). 
Frankl, V. (2010). El hombre en busca de sentido. Barcelona: Herder.

Helman, C. G. (2001). Culture, Health and IIIness. London: Arnold.

Hermann, C. P. (2006). Development and testing of the spiritual needs inventory for patients near to end of life. Oncological Nursing Forum, (33), 737-744. DOI: https://doi.org/10.1188/06.0NF.737-744

Nogués, R. M. (2013). Cerebro y trascendencia. Barcelona: Fragmenta.

OHSJD (Orden Hospitalaria San Juan de Dios). (2012). Carta de identidad de la Orden Hospitalaria de San Juan de Dios. Roma: OHSJD, Roma. Edición revisada (1. ${ }^{\text {a }}$ ed., 2000).

OMS (Organización Mundial de la Salud). (1990). Cancer pain relief and palliative care: report of a WHO expert committee. Geneva.

SECPAL (Sociedad Española de Cuidados Paliativos). (2008). El acompañamiento espiritual en cuidados paliativos. Madrid: SECPAL.

Torralba, F. (2016). Inteligencia espiritual (8. ${ }^{\text {a }}$ ed.). Barcelona: Plataforma Editorial. (1. ${ }^{\text {a }}$ ed., 2010).

Vielva, J. (2007). Ética profesional de la Enfermería. Bilbao: Ed. Descleé de Brouwer.

Yepes, R., y Aranguren, J. (1998). Fundamentos de antropología (3. ${ }^{a}$ ed.). Pamplona: EUNSA. 